\title{
Design of a novel LOX-1 receptor antagonist mimicking the natural substrate
}

\author{
Mattia Falconi ${ }^{\mathrm{a}, *, 1}$, Sarah Ciccone ${ }^{\mathrm{b}, 1}$, Paola D’Arrigo ${ }^{\mathrm{c}, \mathrm{d}}$, Fiorenza Viani ${ }^{\mathrm{e}}$, Roberto Sorge ${ }^{\mathrm{b}}$ \\ Giuseppe Novelli ${ }^{f}$, Patrizia Patrizi ${ }^{g}$, Alessandro Desideri ${ }^{a}$, Silvia Biocca ${ }^{\text {b,* }}$ \\ a Department of Biology, Interuniversity Consortium, National Institute Biostructure and Biosystem (INBB), University of Rome "Tor Vergata", Via della \\ Ricerca Scientifica 1, 00133, Rome, Italy \\ ${ }^{\mathrm{b}}$ Department of Systems Medicine and Center of Biostatistics and Bioinformatics, University of Rome "Tor Vergata", Via Montpellier 1, 00133 Rome, Italy

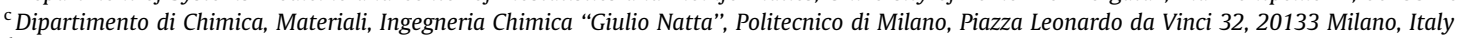 \\ ${ }^{\mathrm{d}}$ The Protein Factory, Centro Interuniversitario di Biotecnologie Proteiche, Politecnico di Milano, ICRM-CNR Milano, via Mancinelli 7, 20131 Milano, Italy \\ e ICRM, CNR, Via Mario Bianco 9, 20131, Milano, Italy \\ ${ }_{\mathrm{f}}$ Department of Biomedicine and Prevention, Genetics Section, University of Rome "Tor Vergata", Via Montpellier 1, 00133, Rome, Italy \\ ${ }^{\mathrm{g}}$ BIOS FIANO S.r.l., Via Tiberina 88, Fiano Romano, Rome, Italy
}

\section{A R T I C L E I N F O}

\section{Article history:}

Received 17 July 2013

Available online 26 July 2013

\section{Keywords:}

LOX-1 inhibitor

ox-LDL

Oxidized phospholipids

Atherosclerosis

Molecular docking

Cell-based assay

\begin{abstract}
A B S T R A C T
The lectin-like oxidized low-density lipoprotein receptor-1 (LOX-1), the major receptor for oxidized lowdensity lipoprotein (ox-LDL) in endothelial cells, is overexpressed in atherosclerotic lesions. LOX-1 specific inhibitors, urgently necessary to reduce the rate of atherosclerotic and inflammation processes, are not yet available. We have designed and synthesized a new modified oxidized phospholipid, named PLAzPC, which plays to small scale the ligand-receptor recognition scheme. Molecular docking simulations confirm that PLAzPC disables the hydrophobic component of the ox-LDL recognition domain and allows the interaction of the L-lysine backbone charged groups with the solvent and with the charged/ polar residues located around the edges of the LOX-1 hydrophobic tunnel. Binding assays, in a cell model system expressing human LOX-1 receptors, confirm that PLAzPC markedly inhibits ox-LDL binding to LOX-1 with higher efficacy compared to previously identified inhibitors.
\end{abstract}

(c) 2013 Elsevier Inc. All rights reserved.

\section{Introduction}

The lectin-like oxidized low-density lipoprotein receptor-1 (LOX-1), located on the surface of vascular endothelial cells, is the major receptor for oxidized low-density lipoprotein (ox-LDL) $[1,2]$. It is a membrane glycoprotein, up-regulated by ox-LDL, expressed also in monocytes/macrophages, smooth muscle cells, dendritic cells and fibroblasts. LOX-1 plays a crucial role during atherogenesis and atherosclerotic plaque formation and is considered a target in a therapeutic perspective [3-5]. It is a disulfidelinked homodimer belonging to the C-type lectin-like receptor family. Deletion analysis has localized ox-LDL recognition activity to the highly conserved C-type lectin-like domain (CTLD) of

Abbreviations: CTLD, C-type lectin-like domain; Dil, 1,1'-dioctadecyl-3,3,3', 3'-tetramethyllindocarbocyanine perchlorate; HOBt, 1-hydroxybenzotriazole; LDL, low-density lipoprotein; LOX-1, lectin-like oxidized low-density lipoprotein receptor-1; ox-LDL, oxidized low-density lipoprotein; SRB, sulforhodamine B.

* Corresponding authors.

E-mail addresses: falconi@uniroma2.it (M. Falconi), biocca@med.uniroma2.it (S. Biocca).

1 These two authors contributed equally to this work.
LOX-1 [6]. The crystal structures of the human CTLD have largely contributed to elucidate the molecular recognition and binding mechanisms of LOX-1 $[7,8]$.

The human LOX-1 CTLD forms a heart-shaped homodimer with an inter-chain disulfide bond at Cys140, not present in sequences of other species. Mutation of Cys140 does not affect the ox-LDLbinding activity $[9,10]$ and does not destabilize the dimeric form of the ligand-binding domain [10]. The particular dimeric association of CTLD in human LOX-1 generates a tunnel which completely crosses the dimer with a diameter of 7.0-8.0 $\AA$, except for a first constriction caused by the side chains of Ile149 of the two subunits and a second constriction caused by Tyr 197 of subunit A, that restrict the opening to $4.0 \AA$ [7]. In the central part, just below the tunnel, the residue Trp150 is completely conserved among the LOX-1 orthologs and 2 salt bridges and 7 inter-chain hydrogen bonds contribute to the stabilization of LOX-1 dimer arrangement [7]. On the top, human LOX-1 displays a linear arrangement of positively charged residues, crossing the dimer surface of CTLD, called basic spine $[7,8]$ that has been proposed to provide an appropriate platform for the interaction and the electrostatic recognition of the amphipathic $\alpha$-helices located on the ox-LDL surface [7]. 
Mutations of the basic spine arginines of the dimer interface residues Trp150 and Lys167, which alter the basic spine structure $[11,12]$, markedly reduces LOX-1 binding activity $[8,10,13]$. Over the CTLD dimer surface the entrance of the long hydrophobic tunnel is surrounded by a quasi-conical surface where hydrophilic and hydrophobic patches are scattered. Mutations of residues located in a position that obstructs the tunnel and the presence of phospholipids that can interact with residues in the tunnel, significantly prevent the binding ability of LOX-1 receptor to ox-LDL [14].

Starting from these observations, a rational approach to design a selective receptor antagonist introducing new recognition elements has been followed. In this work we present the synthesis of a new L-lysine condensated phospholipid compound, named PLAzPC, which is able to disengage the recognition between oxLDL and LOX-1. Binding assays, in a cell model system expressing human LOX-1 receptors, confirm that acute exposure of PLAzPC results in a marked reduction of ox-LDL bound to LOX-1.

\section{Materials and methods}

\subsection{Molecular docking procedure}

Protein-ligand molecular docking, a computational technique that predicts the putative binding site of a ligand on a receptor taking into account both geometrical and electrostatic match contributions, has been used to predict the complexes between the C-type lectin like domain (CTLD) of the human LOX-1 receptor with the phospholipids: PAzPC, HAzPC, PGPC, PONPC, POVPC and the newly synthetized compound PLAzPC. The phospholipids are prepared using the program SYBYL 6.0 (Tripos Inc., 1699, South Hanley Road St. Louis, Missouri 63144, USA). The polar hydrogens are added to the protein and the phospholipids. The protein-ligand docking has been executed with the AutoDock Vina 1.1.2 program [15], using the AutoDock/Vina PyMOL plugin (http://wwwuser.gwdg.de/ dseelig/adplugin.html) [16,17]. For more details see Supplementary data 1 .

\subsection{Synthesis of PLAzPC}

To obtain 1-palmitoyl-2-(9-(5-t-Boc-amino-5-carboxypentylamino)-9-oxononanoyl)-sn-glycero-3-phosphocholine (PtBocLAzPC-(2)) a solution of 1-palmitoyl-2-azelaoyl-sn-glycero-3-phosphocholine (Avanti Polar Lipids, PAzPC (1), $0.0375 \mathrm{mmol}, 25 \mathrm{mg}$ ) dissolved in DMSO (2.5 mL), 1-hydroxybenzotriazole (HOBt) $(0.15 \mathrm{mmol}$, $20.3 \mathrm{mg}$ ) was added at room temperature (Fig. 1). The clean solution was stirred for $1 \mathrm{~h}$ at r.t., then cooled at $0{ }^{\circ} \mathrm{C}$ (ice-water bath) and neat 1-ethyl-3-(3-dimethylaminopropyl) carbodiimide hydrochloride (EDC.HCl, $0.15 \mathrm{mmol}, 28.7 \mathrm{mg}$ ) was added. Then $\mathrm{N}-\mathrm{t}$-BocLysine $(0.0375 \mathrm{mmol}, 9.25 \mathrm{mg})$ and sym-collidine $(0.041 \mathrm{mmol}$, $5.83 \mathrm{~mL})$ in $\mathrm{CH}_{2} \mathrm{Cl}_{2}(2.0 \mathrm{~mL})$ were added under stirring at the same temperature. The ice-water bath was removed and the temperature was allowed to reach r.t. Stirring was continued for 5 days while the reaction was monitored by TLC both in $\mathrm{CH}_{2} \mathrm{Cl}_{2} / \mathrm{MeOH} /$ $\mathrm{NH}_{3}$ 85:15:2.5 and in toluene/MeOH/Et $3{ }_{3} \mathrm{~N}$ 85:15:5. The workingup procedure was as follows: water $(2 \mathrm{~mL})$ was added and the organics were extracted in chloroform $(3 \times 2 \mathrm{~mL})$. The combined organic layers were dried over anhydrous sodium sulfate, filtered and the solvent was removed under reduced pressure. The residue was purified by flash chromatography using a gradient elution: pure $\mathrm{CHCl}_{3}(20 \mathrm{~mL}), \mathrm{CHCl}_{3} / \mathrm{MeOH} 68: 32(20 \mathrm{~mL}), \mathrm{CHCl}_{3} / \mathrm{MeOH} /$ $\mathrm{NH}_{3}$ 68:32:2.5 (20 mL), $\mathrm{MeOH} / \mathrm{NH}_{3}$ 100:2.5 (20 mL). The product (2) was isolated in $27 \%$ yield $(9 \mathrm{mg}): R_{\mathrm{F}}=0.25\left(\mathrm{CHCl}_{3} / \mathrm{MeOH} / \mathrm{NH}_{3}\right.$ 68:32:2.5); $[\alpha]_{\mathrm{D}}^{20}=+2.49($ c $1.3, \mathrm{MeOH}),[\alpha]_{\mathrm{D}}^{20}=+12.22$ (c 0.9 , DMSO); Mass $(m / z)$ : $894.6[\mathrm{M}+1]^{+}, 892.6[\mathrm{M}-1]^{+} ;{ }^{1} \mathrm{H} \mathrm{NMR}$ $\left(\mathrm{CDCl}_{3}+\mathrm{CD}_{3} \mathrm{OD}\right): \delta 0.90$ (brt, $\left.3 \mathrm{H}\right), 1.25-1.40$ (m, 32H), 1.45 (brs,
9H), $1.51(\mathrm{~m}, 2 \mathrm{H}), 1.55-1.70(\mathrm{~m}, 4 \mathrm{H}), 1.80(\mathrm{~m} .2 \mathrm{H}), 2.15-2.40(\mathrm{~m}$, $6 \mathrm{H}), 3.15(\mathrm{~m}, 2 \mathrm{H}), 3.24$ (brs, $9 \mathrm{H}), 3.66(\mathrm{~m}, 2 \mathrm{H}), 4.11$ and $4.35(\mathrm{~m}$, $2 \mathrm{H}), 4.20$ and $4.44(\mathrm{~m}, 2 \mathrm{H}), 4.32(\mathrm{~m}, 2 \mathrm{H}), 4.55(\mathrm{~m}, 1 \mathrm{H}), 5.25(\mathrm{~m}$, 1H). To obtain 1-palmitoyl-2-(9-(5-amino-5-carboxypentylamino)-9-oxononanoyl)-sn-glycero-3-phosphocholine (PLAzPC, (3)) a $1: 4 \mathrm{CF}_{3} \mathrm{COOH} / \mathrm{CH}_{2} \mathrm{Cl}_{2}$ mixture $(0.5 \mathrm{~mL})$ was added to a solution of $\mathrm{P}^{\mathrm{t}}$ BocLAzPC (2) $(0.0101 \mathrm{mmol}, 9 \mathrm{mg})$ in $\mathrm{CH}_{2} \mathrm{Cl}_{2}(0.2 \mathrm{~mL})$ at r.t. The mixture was stirred for $5 \mathrm{~h}$. Cyclohexane $(3 \times 0.5 \mathrm{~mL})$ was added to remove $\mathrm{CF}_{3} \mathrm{COOH}$ under reduced pressure. The row residue was dried under vacuum and the desired compound (3) was isolated and analyzed without further purification as trifluoroacetic salt.

$[\alpha]_{\mathrm{D}}^{20}=+2.02\left(\mathrm{c} 0.5, \mathrm{CHCl}_{3}\right),[\alpha]_{\mathrm{D}}^{20}=+3.83$ (c 1.1, MeOH); Mass $(\mathrm{m} / \mathrm{z}): 794.9[\mathrm{M}+\mathrm{H}]^{+}, 816.9[\mathrm{M}+\mathrm{Na}]^{+} ; 792.7[\mathrm{M}-\mathrm{H}]^{-} ; \mathrm{M} / \mathrm{M}: 777.8$ $\left[\mathrm{M}+\mathrm{H}-\mathrm{NH}_{3}\right]^{+} ; 735.8\left[\mathrm{M}+\mathrm{H}-\left(\mathrm{CH}_{3}\right)_{3} \mathrm{~N}\right]^{+} ; 733.7\left[\mathrm{M}-\mathrm{H}-\left(\mathrm{CH}_{3}\right)_{3} \mathrm{~N}\right]^{-} ;{ }^{1} \mathrm{H}$ $\operatorname{NMR}\left(\mathrm{CD}_{3} \mathrm{OD}\right) \delta: 0.90(\mathrm{t}, 3 \mathrm{H}), 1.25-1.40(\mathrm{~m}, 32 \mathrm{H}), 1.50(\mathrm{~m}, 2 \mathrm{H})$, 1.55-1.72 (m, 6H), $1.76(\mathrm{~m}, 2 \mathrm{H}), 2.10-2.40(\mathrm{~m}, 6 \mathrm{H}), 3.22(\mathrm{~m}, 2 \mathrm{H})$, 3.25 (brs, $9 \mathrm{H}), 3.49(\mathrm{~m}, 1 \mathrm{H}), 3.70(\mathrm{~m}, 2 \mathrm{H}), 4.13$ and $4.36(\mathrm{~m}, 2 \mathrm{H})$, 4.18 and $4.43(\mathrm{~m}, 2 \mathrm{H}), 4.30(\mathrm{~m}, 2 \mathrm{H}), 5.22(\mathrm{~m}, 1 \mathrm{H})$.

\subsection{DNA construct}

For the expression in mammalian cells, human LOX-1 was subcloned into pEF/V5-His vectors (Invitrogen, Inchinnan, Paisley, UK), as previously described [18].

\subsection{Cell cultures and transfection}

COS cells were transiently transfected with JetPEI (Polyplus Transfection, Illkirch, France), following the manufacturer's instructions, with a DNA/transfectant reagent ratio $(\mathrm{w} / \mathrm{v})$ of $1: 2$.

\subsection{Immunofluorescence}

Cell membrane immunofluorescence was carried out as described [19] using anti-V5 IgG (Invitrogen, Inchinnan, Paisley, UK) as primary antibody and Rhodamine Red-X-conjugated AffiniPure donkey anti-mouse IgG (Jackson Immunoresearch, West Grove, PA, USA) as secondary antibody. Samples were examined with a DMRA Leica fluorescence microscope, equipped with CCD camera.

\section{6. $0 x-L D L$ preparation, labeling and binding to LOX-1 receptors}

Human LDL was prepared from fresh healthy normolipidemic plasma of volunteers by ultracentrifugation [20]. Oxidation was performed as described [21]. ox-LDL was labeled with 1,1'-dioctadecyl-3,3,3',3'-tetramethyllindocarbocyanine perchlorate (DiI, Invitrogen, Inchinnan, Paisley, UK) as previously described to give a fluorescent Dil-ox-LDL $[18,22]$.

COS cells were plated in 48 multiwell at $70 \%$ confluence, transfected with LOX-1-V5 plasmid. Binding assay was performed $24 \mathrm{~h}$ after transfection as described [23]. COS cells were incubated 30 min at $4{ }^{\circ} \mathrm{C}$ with different inhibitors. Cells were then incubated with (DiI)-labeled ox-LDL $10 \mu \mathrm{g} / \mathrm{ml}$ in the presence of inhibitors on ice for $1 \mathrm{~h}$. Quantification of bound Dil-ox-LDL was assayed by Dil extraction in isopropanol [22] and fluorescence determined in a Perkin Elmer spectrofluorometer. Dil-ox-LDL binding was also performed in transfected COS cells plated in glass coverslips. After $1 \mathrm{~h}$ incubation with (DiI)-labeled ox-LDL cells were fixed in paraformaldehyde and stained with Hoescht 33342 (Sigma) before mounting. Dil-ox-LDL positive cells were counted and the percentage of positive cells with respect of all Hoescht-positive nuclei was calculated. At least 120-150 cells were counted and checked for their Dil-ox-LDL positivity for each coverslip. For a description of the cell viability and cytotoxicity assay see the Supplementary data 2 . 

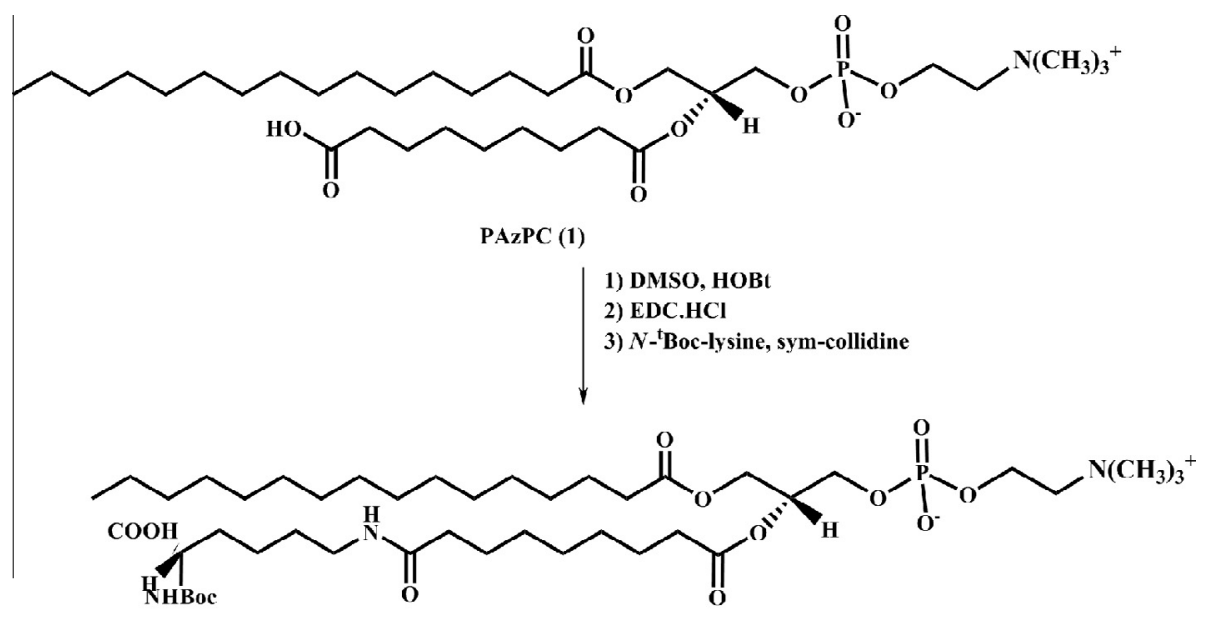

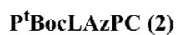

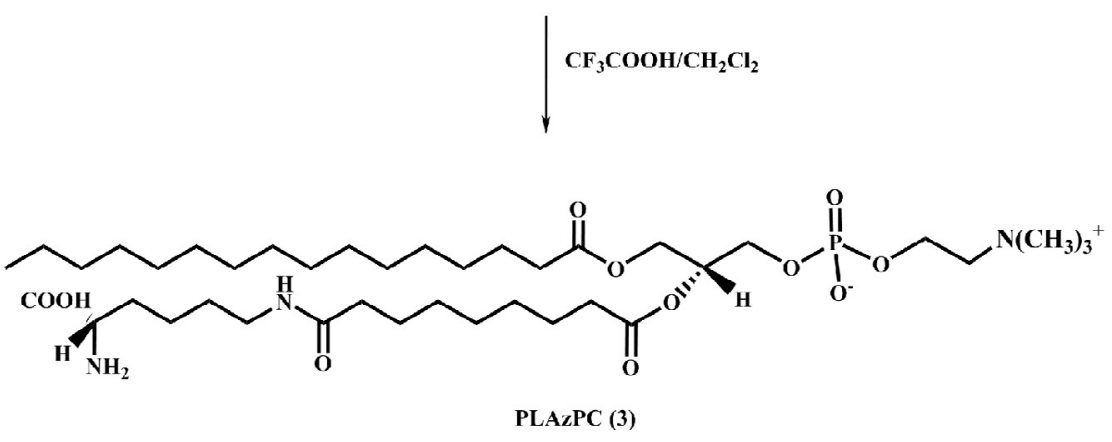

Fig. 1. Synthesis of 1-palmitoyl-2-(9-(5-amino-5-carboxypentylamino)-9-oxononanoyl)-sn-glycero-3-phosphocholine (PLAzPC).

\subsection{Statistical data analysis}

All data were inserted into an Excel database (Microsoft, Redmond, Washington, USA) and analyzed with the Statistical Package for the Social Sciences Windows version 15.0 (SPSS, Chicago, Illinois, USA). Descriptive statistics used for continuous variables was the average \pm standard deviation. Normality assumptions were demonstrated with histograms and the Kolmogorov-Smirnov test. Comparison among groups was performed using one-way ANOVA with multiple comparisons by Bonferroni test. A $p$ value $<0.05$ was considered statistically significant.

\section{Results and discussion}

\subsection{Structural analysis, synthesis and in vivo inhibitory activity of PLAzPC}

On the basis of mutagenesis experiments carried out nearby the hydrophobic tunnel [14], supported by structural observations on the LOX-1 recognition domain, we have designed a new LOX-1 receptor antagonist mimicking one of the phospholipid adducts that are formed by oxidation on the apoB100 surface [21]. The compound incorporates a dual function to interfere with the recognition components of the receptor: (i) an hydrophobic moiety, required to interact and obstruct the CTLD hydrophobic tunnel involved in the substrate binding [14] and (ii) an electrostatic and flexible moiety, that has been introduced to install interactions with water and charged residues located in the proximity of the tunnel border.

The new compound, 1-palmitoyl-2-(9-(5-amino-5-carboxypentylamino)-9-oxononanoyl)-sn-glycero-3-phosphocholine, named
PLAzPC, has been synthesized following the scheme depicted in Fig. 1. The 1-palmitoyl-2-azelaoyl-sn-glycero-3-phosphocholine (PAzPC) phospholipid has been altered adding two hydrophilic centers in the 2-azelaoyl tail. For this purpose a lysine derivative, namely the $N_{\alpha}$-(tert-butoxycarbonyl)-L-lysine, has been condensated to the free carboxylic acid function present in the $s n-2$ position of PAzPC (Fig. 1), providing, after hydrolysis of the lysine protecting group, the new phospholipid PLAzPC (3 in Fig. 1).

Protein-ligand molecular docking, a computational technique that predicts the putative binding site of a ligand on a receptor, taking into account both geometrical and electrostatic match contributions, has been used to support the formation of a complex between the CTLD and the chosen drugs. The docking simulations indicate that PLAzPC is able to fill the hydrophobic tunnel of the receptor but is also stabilized by electrostatic interactions with water and other residues (Supplementary data 3). In PLAzPC the targeted chemical modification adds an essential electrostatic contribution that stabilizes its binding into the tunnel. Thus, the LOX-1 residues Gln193 and Ser198, in one subunit, and Ser160 in the other interact with hydrophilic PLAzPC spots (Fig. 2 and Supplementary data 3, Fig. S3 for details). Moreover, the charged amino-acid group of the condensated L-lysine is placed toward the solvent, shielding one of the two entries of the filled tunnel, while the other entry is plugged by the charged phosphocholine. This double PLAzPC-solvent interface critically stabilizes the ligand into the tunnel, preventing water from interfering with the hydrophobic binding.

As a benchmark for the novel functionality introduced in the PLAzPC inhibitor, we have compared PLAzPC recognition capability with that of different oxidized phospholipids, whose structure denotes potentiality for the interaction with the hydrophobic tunnel [14]. In 

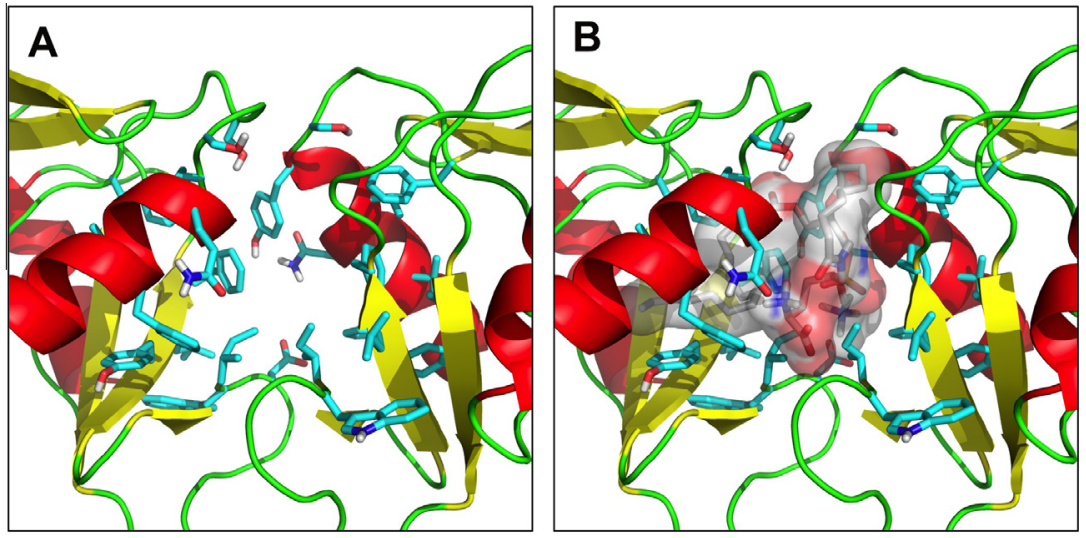

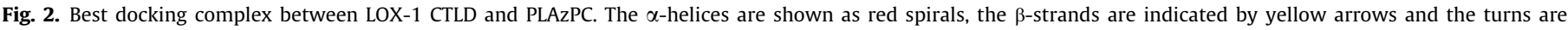

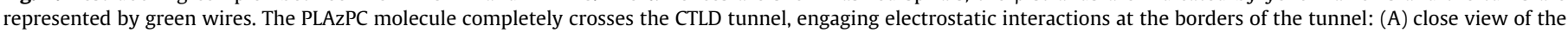

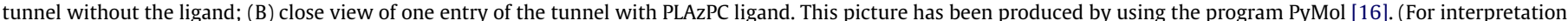
of the references to color in this figure legend, the reader is referred to the web version of this article.)

particular, we have analyzed the following compounds: 1-palmitoyl2-glutaryl-sn-glycero-3-phosphocholine (PGPC), 1-O-hexadecyl-2-azelaoyl-sn-glycero-3-phosphocholine (HAzPC), 1-palmitoyl-2-azelaoylsn-glycero-3-phosphocholine (PAzPC) (Fig. S2). The docking binding energy, which varies between -8.2 of PGPC and HAzPC to -8.6 of $\mathrm{PAzPC}$ reaches the value of $-9.1 \mathrm{kcal} / \mathrm{mol}$ in the case of the newly synthetized PLAzPC.

To verify the in vivo LOX-1 inhibitory activity of PLAzPC, we have set up a cell model expressing high level of human LOX-1 receptors. This was achieved by transiently transfecting full length human LOX-1-V5 DNA into COS fibroblasts, as previously described [23]. Cell surface expression of LOX-1 receptors was demonstrated by anti-V5 binding and immunofluorescence. This cell model allows a high transfection efficiency (about $40 \%$ of transfected cells), a very high expression level of LOX-1 receptors and very high ox-LDL binding (Fig. 3) and uptake [23]. Considering that LOX1 is an inducible gene and it is highly expressed under stress conditions, this cell system is appropriate to recapitulate the in vivo characteristics. In order to monitor the LOX-1-ox-LDL binding we have used the highly fluorescent lipophilic dye $1,1^{\prime}$-dioctadecyl$3,3,3^{\prime}, 3^{\prime}$-tetramethyllindocarbocyanine perchlorate (DiI), that diffuses into the hydrophobic portion of the LDL complex without affecting the LDL-specific binding of the apoprotein [22]. We measured the capacity to displace the binding of fluorescent DiI-ox-LDL to LOX-1 of PLAzPC and, for comparison, that of the oxidized phospholipids PGPC, HAzPC, PAzPC, using two different approaches: a) by counting positive DiI-ox-LDL fluorescent cells in the absence and in the presence of different concentration of the indicated compound and b) by quantitation of bound DiI-ox-LDL with its extraction from stained cells with isopropanol and spectrofluorometric analysis. The binding specificity was confirmed by incubating with 100-fold excess of cold ox-LDL which completely abolishes Dil-ox-LDL binding to LOX-1 (not shown). Comparison among groups was performed using one-way ANOVA with multiple comparisons by Bonferroni test. Cell viability and cytotoxicity were measured by the sulforhodamine B assay (SRB) as described in Supplementary data 2. No effects on cell viability are observed when cells are incubated with different doses (ranging from 2 to $20 \mu \mathrm{M}$ ) of oxidized phospholipids for at least 5 days. A moderate toxicity is observed after 2 days of incubation with a concentration higher than $40 \mu \mathrm{M}$ (Fig. S1).

As it can be seen in Fig. 3, a marked reduction of Dil-ox-LDL positive cells was obtained incubating cells with PLAzPC. The statistical analysis indicates that, at $5 \mu \mathrm{M}$ concentration, the percentage decrease of ox-LDL positive cells was $59 \pm 8 \%$ vs controls $(p<0.01)$. The quantification of bound Dil-ox-LDL through spectrofluorometric analysis is shown in Fig. 4. At $5 \mu \mathrm{M}$ concentration, PLAzPC leads to $63 \pm 6 \%$ reduction of bound fluorescence, while HAzPC, PAzPC and PGPC are less effective and lead to $16 \pm 8 \%$, $39 \pm 9 \%$ and $11 \pm 9 \%$ reduction of bound Dil-ox-LDL vs control, respectively. Importantly, when we analyzed the intracellular uptake of fluorescent ox-LDL by incubating transfected cells with Dil-ox-LDL at $37^{\circ} \mathrm{C}$ for $1 \mathrm{~h}$, we observed a very similar rate of inhibition (data not shown).

Notably, in PLAzPC treated cells, the fluorescence intensity does not uniformly decreases (Fig. 3). Transient transfection in COS cells
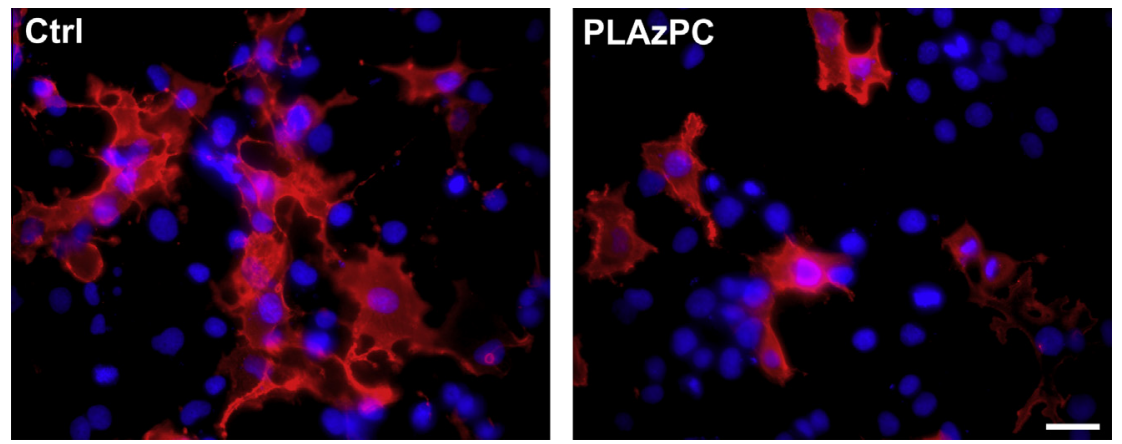

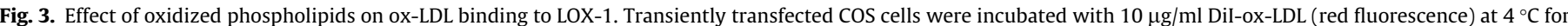

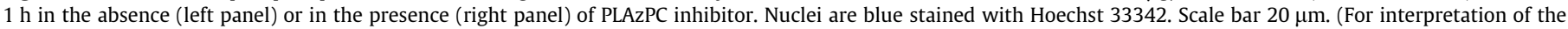
references to colour in this figure legend, the reader is referred to the web version of this article.) 

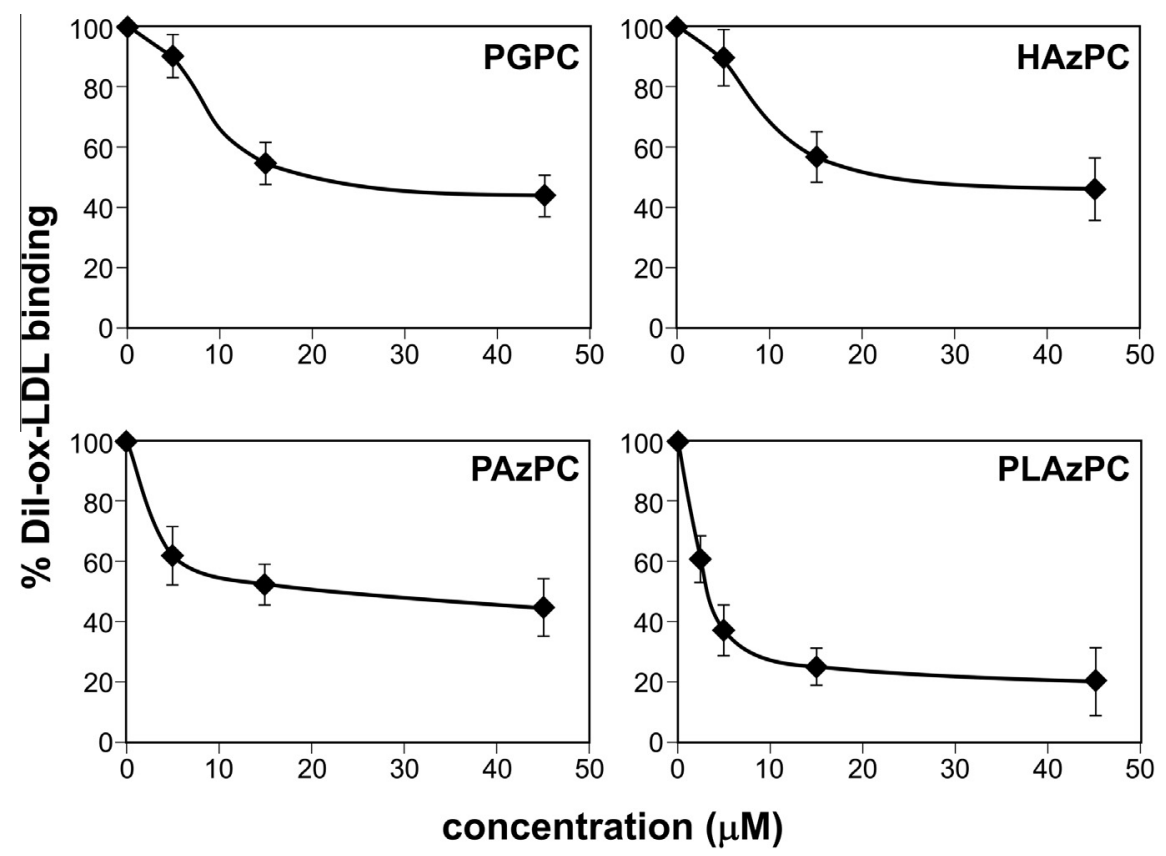

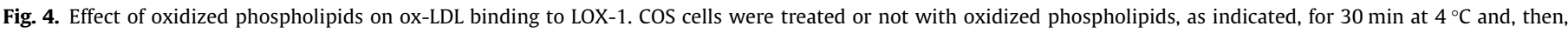

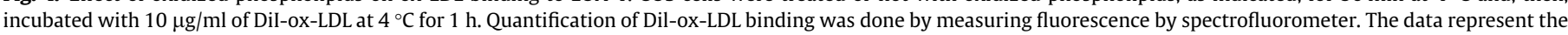
mean \pm standard deviation calculated from four separate experiments.

results in a very high LOX-1 expression variability. We suggest that only cells with lower expression level are "switched off", while those with higher LOX-1 level are still able to bind enough Dilox-LDL molecules to appear fluorescent. Moreover, a residual amount of bound fluorescent Dil-ox-LDL is not displaced even in the presence of high concentration of oxidized phospholipids (Fig. 4). A possible structural explanation of this finding is that while the hydrophobic component of the recognition, mainly due to the tunnel, is completely disabled by the different oxidized phospholipids, the electrostatic attraction of the apoB100 protein located on the ox-LDL surface and the LOX-1 basic spine is still active. In particular, although free charged PLAzPCs could also engage interactions with the basic spine arginines (through the carboxyl of the condensated L-lysine), the electrostatic repulsion due to a close positive $\alpha$-ammonium group and the positive charge of the choline group, make this hypothesis unlikely. It is also important to underline that multimerization and cluster organization in plasma membrane, that are important requisites for LOX-1 activity [10,23,24], may interfere with the binding of the inhibitor and can influencethe ox-LDL binding affinity. In fact, clusters develop regardless of the presence of ox-LDL and their formation is LOX-1 concentration-dependent [25]. It is worth mentioning that the residual oxLDL binding activity is totally displaced when a 100 times excess of unlabeled ox-LDL is added to the binding solution.

The in vivo results and the in silico molecular docking structural analysis indicate that PLAzPC is a strong inhibitor of LOX-1 receptor. This compound completely disables the hydrophobic component of the ox-LDL recognition domain mainly due to the tunnel and is also stabilized by the interactions of the L-lysine backbone charged groups. These groups, extending from the azelaoyl chain, interact both with the solvent and with the charged/polar residues located around the edges of the tunnel $[7,8]$. From data shown in Fig. 4, in the case of PLAzPC, this mechanism leads to approximately $80 \%$ inhibition of ox-LDL binding to LOX-1.

As mentioned before, we have compared PLAzPC recognition capability with that of different oxidized phospholipids (PGPC, HAzPC and PAzPC), which have been previously studied [14]. Differences between experimental protocols may justify small discrepancy in inhibitory efficiency evaluation. Francone et al. evaluated the ox-LDL binding through an in vitro assay by isolation of plasma membranes from stably transfected cells, while we use a binding assay in transiently transfected living cells, which leads to a higher LOX-1 receptor expression efficiency.

In conclusion the structural approach followed to synthesize a novel antagonist of LOX-1 receptor gives novel and interesting insights about the molecular mechanisms of inhibition of the LOX-1ox-LDL binding and indicates that PLAzPC represents a lead compound for the development of selective therapeutic molecules with high inhibitory effect.

\section{Acknowledgments}

This work was partially supported by FILAS for the "FineSTRE project" and TECNO.TIB.E.R.I.S. for the "MOLBLOX" project. We are also very grateful to Graziano Bonelli for his expert graphical assistance.

\section{Appendix A. Supplementary data}

Supplementary data associated with this article can be found, in the online version, at http://dx.doi.org/10.1016/j.bbrc.2013.07.073.

\section{References}

[1] T. Sawamura, N. Kume, T. Aoyama, H. Moriwaki, H. Hoshikawa, Y. Aiba, T. Tanaka, S. Miwa, Y. Katsura, T. Kita, T. Masaki, An endothelial receptor for oxidized low-density lipoprotein, Nature 386 (1997) 73-77.

[2] M. Chen, T. Masaki, T. Sawamura, LOX-1, the receptor for oxidized low-density lipoprotein identified from endothelial cells: implications in endothelial dysfunction and atherosclerosis, Pharmacol. Ther. 95 (2002) 89-100.

[3] J.L. Mehta, J. Chen, P.L. Hermonat, F. Romeo, G. Novelli, Lectin-like, oxidized low-density lipoprotein receptor-1 (LOX-1): a critical player in the development of atherosclerosis and related disorders, Cardiovasc. Res. 69 (2006) 36-45.

[4] S. Dunn, R.S. Vohra, J.E. Murphy, S. Homer-Vanniasinkam, J.H. Walker, S. Ponnambalam, The lectin-like oxidized low-density-lipoprotein receptor: a pro-inflammatory factor in vascular disease, Biochem. J. 409 (2008) 349-355 
[5] S. Mitra, A. Deshmukh, R. Sachdeva, J. Lu, J.L. Mehta, Oxidized low-density lipoprotein and atherosclerosis implications in antioxidant therapy, Am. J. Med. Sci. 342 (2011) 135-142.

[6] A.N. Zelensky, J.E. Gready, The C-type lectin-like domain superfamily, FEBS J. 272 (2005) 6179-6217.

[7] H. Park, F.G. Adsit, J.C. Boyington, The 1.4 angstrom crystal structure of the human oxidized low density lipoprotein receptor LOX-1, J. Biol. Chem. 280 (2005) 13593-13599.

[8] I. Ohki, T. Ishigaki, T. Oyama, S. Matsunaga, Q. Xie, M. Ohnishi-Kameyama, T. Murata, D. Tsuchiya, S. Machida, K. Morikawa, S. Tate, Crystal structure of human lectin-like, oxidized low-density lipoprotein receptor 1 ligand binding domain and its ligand recognition mode to OxLDL, Structure 13 (2005) 905917.

[9] Q. Xie, S. Matsunaga, S. Niimi, S. Ogawa, K. Tokuyasu, Y. Sakakibara, S. Machida, Human lectin-like oxidized low-density lipoprotein receptor-1 functions as a dimer in living cells, DNA Cell Biol. 23 (2004) 111-117.

[10] I. Ohki, H. Amida, R. Yamada, M. Sugihara, T. Ishigaki, S. Tate, Surface plasmon resonance study on functional significance of clustered organization of lectinlike oxidized LDL receptor (LOX-1), Biochim. Biophys. Acta 2011 (1814) 345 354.

[11] M. Falconi, S. Biocca, G. Novelli, A. Desideri, Molecular dynamics simulation of human LOX-1 provides an explanation for the lack of OxLDL binding to the Trp150Ala mutant, BMC Struct. Biol. 7 (2007) 73.

[12] S. Nakano, M. Sugihara, R. Yamada, K. Katayanagi, S. Tate, Structural implication for the impaired binding of W150A mutant LOX-1 to oxidized low density lipoprotein, OxLDL, Biochim. Biophys. Acta 2012 (1824) 739-749.

[13] S. Biocca, M. Falconi, I. Filesi, F. Baldini, L. Vecchione, R. Mango, F. Romeo, G. Federici, A. Desideri, G. Novelli, Functional analysis and molecular dynamics simulation of LOX-1 K167N polymorphism reveal alteration of receptor activity, PLoS One 4 (2009) e4648.

[14] O.L. Francone, M. Tu, L.J. Royer, J. Zhu, K. Stevens, J.J. Oleynek, Z. Lin, L. Shelley, T. Sand, Y. Luo, C.D. Kane, The hydrophobic tunnel present in LOX-1 is essentia for oxidized LDL recognition and binding, J. Lipid Res. 50 (2009) 546-555.
[15] O. Trott, A.J. Olson, AutoDock Vina: improving the speed and accuracy of docking with a new scoring function, efficient optimization, and multithreading, J. Comput. Chem. 31 (2010) 455-461.

[16] W.L. DeLano, The PyMOL Molecular Graphics System, DeLano Scientific, San Carlos, CA, USA, 2002.

[17] D. Seeliger, B.L. de Groot, Ligand docking and binding site analysis with PyMOL and Autodock/Vina, J. Comput. Aided Mol. Des. 24 (2010) 417-422.

[18] S. Biocca, I. Filesi, R. Mango, L. Maggiore, F. Baldini, L. Vecchione, A. Viola, G. Citro, G. Federici, F. Romeo, G. Novelli, The splice variant LOXIN inhibits LOX-1 receptor function through hetero-oligomerization, J. Mol. Cell. Cardiol. 44 (2008) 561-570.

[19] A. Cardinale, I. Filesi, V. Vetrugno, M. Pocchiari, M.S. Sy, S. Biocca, Trapping prion protein in the endoplasmic reticulum impairs PrPC maturation and prevents PrPSc accumulation, J. Biol. Chem. 280 (2005) 685-694.

[20] W. Sattler, P. Bone, R. Stocker, Isolation of human VLDL, LDL, HDL and two HDL subclasses in the TL-100 tabletop centrifuge using the TLA-100.4 rotor, Technical Information, Beckman, 1992.

[21] K.L. Gillotte, S. Horkko, J.L. Witztum, D. Steinberg, Oxidized phospholipids, linked to apolipoprotein B of oxidized LDL, are ligands for macrophage scavenger receptors, J. Lipid Res. 41 (2000) 824-833.

[22] Z.F. Stephan, E.C. Yurachek, Rapid fluorometric assay of LDL receptor activity by Dil-labeled LDL, J. Lipid Res. 34 (1993) 325-330.

[23] S. Matarazzo, M.C. Quitadamo, R. Mango, S. Ciccone, G. Novelli, S. Biocca, Cholesterol-lowering drugs inhibit lectin-like oxidized low-density lipoprotein-1 receptor function by membrane raft disruption, Mol. Pharmacol. 82 (2012) 246-254.

[24] W. Cao, V. Calabro, A. Root, G. Yan, K. Lam, S. Olland, J. Sanford, A. Robak, R Zollner, Z. Lu, M. Ait-Zahra, R. Agostinelli, L. Tchistiakova, D. Gill, D. Harnish, J. Paulsen, H.H. Shih, Oligomerization is required for the activity of recombinant soluble LOX-1, FEBS J. 276 (2009) 4909-4920.

[25] S. Matsunaga, Q. Xie, M. Kumano, S. Niimi, K. Sekizawa, Y. Sakakibara, S. Komba, S. Machida, Lectin-like oxidized low-density lipoprotein receptor (LOX-1) functions as an oligomer and oligomerization is dependent on receptor density, Exp. Cell Res. 313 (2007) 1203-1214. 